\title{
Dynamics of the Rumor-Spreading Model with Control Mechanism in Complex Network
}

\author{
Wei Zhang, ${ }^{1}$ Hongyong Deng $\mathbb{D}^{1},{ }^{1}$ Xingmei $\mathrm{Li}^{1}{ }^{1}$ and Huan Liu ${ }^{2}$ \\ ${ }^{1}$ School of Data Science and Information Engineering, Guizhou Minzu University, Guiyang 550025, China \\ ${ }^{2}$ College of Tourism and Environment, Zhangjiakou University, Zhangjiakou 075000, China \\ Correspondence should be addressed to Hongyong Deng; dhycel@vip.163.com
}

Received 29 September 2021; Accepted 14 December 2021; Published 12 January 2022

Academic Editor: Kenan Yildirim

Copyright (c) 2022 Wei Zhang et al. This is an open access article distributed under the Creative Commons Attribution License, which permits unrestricted use, distribution, and reproduction in any medium, provided the original work is properly cited.

The spread of rumors has a great impact on social order, people's psychology, and life. In recent years, the application of rumorspreading models in complex networks has received extensive attention. Taking the management and control of rumors by relevant departments in real life into account, the SIDRQ rumor-spreading model that combines forgetting mechanism, immune mechanism, and suspicion mechanism and guides on a uniform network is established in this paper. Then, the basic reproductive number of the system and the unique existence of the solution are discussed, and the stability of the system is analyzed using the basic reproductive number, Lyapunov function, and Lienard and Chipart theorem; furthermore, the basic reproductive number may not be able to deduce the stability of the system and a counterexample is given. Finally, the influence of different parameters on the spread of rumors is studied, and the validity of the theoretical results is verified.

\section{Introduction}

Rumors refer to unconfirmed information and are spread in certain ways. The rumor-spreading model can be traced back to the 1960s. Daley and Kendall's [1] "Stochastic Rumors" paper borrowed the infectious disease model and proposed the rumor propagation model for the first time.

Later researchers called the rumor-spreading model in the name of the author for the D-K model. The D-K model analyzes the problem of rumors based on a random process and divides people in the process of spreading rumors into three categories: people who have never heard of rumors, people who spread rumors, and people who have heard rumors but did not spread them. Mak [2] later modified the $\mathrm{D}-\mathrm{K}$ model and proposed the $\mathrm{M}-\mathrm{K}$ model, in which rumors spread directly with other people through the communicator. It is found that the D-K model and the M-K model are only applicable to rumor propagation models in small-scale social networks, but social networks in daily life are large in scale and have the characteristics of small world and scalefree. Zanette studied the spread of rumors on the WS small world network considering the topology $[3,4]$. Moreno et al.
[5] studied the $\mathrm{M}-\mathrm{K}$ model on scale-free networks and recorded the interaction between the network topology and the rules of the $\mathrm{M}-\mathrm{K}$ model.

By combining the M-K model with the SIR model, Nekovee et al. [6] further studied the rumor propagation state of complex networks in general and took the forgetting mechanism into account and proposed a rumor propagation model with immune mechanism and forgetting mechanism. Wei [7] introduced the forgetting and memory mechanism in the SI model and analyzed the influence of information dissemination on the BA scale-free network by means of numerical simulation, and the results showed that the forgetting memory mechanism may lead to the termination of dissemination. According to the characteristics that people will forget and then remember for a short time, they become communicators when they are remembered. Zhao et al. $[8,9]$ call this kind of people who temporarily forgot to spread rumors when they encounter a communicator and start spreading rumors as a hibernator, call this mechanism a memory mechanism, and propose a hibernator, immune mechanism, and forgetting mechanism. The SIHR rumor dissemination model based on the memory mechanism uses 
the mean field theory to analyze the dissemination behavior of SIHR on uniform and nonuniform networks. In real life, a person is likely to forget the current possession of information and also recall the features of the forgotten information.

Then, Zhao et al. [10] proposed a SIRaRu model with the speed of forgetting based on people's characteristics. The verification results show that the network topology has a significant impact on the rumor propagation, and the final size of the rumor is greatly affected by the forgetting rate. From the analysis of the research work on the rumorspreading model, it can be seen that compared with infectious diseases, rumor spreading has specific complex network characteristics. The dissemination of information usually follows social relations. The rapid development of the Internet and the network society has made this phenomenon even more prominent. Due to the diversity and complexity of network information dissemination models, people are constantly applying infectious disease models to rumors dissemination.

Mathematical models play an important role in the study of the spread of rumors, and the spread of rumors in society can be seen as a network communication behavior that obeys a certain law. Therefore, studying the influence of network structure on communication behavior has naturally become a very important topic.

In addition, most previous works assume that rumors spread in a closed system and the population size in OSNs (Open Systems Network Support) is constant. Consequently, these models can only simulate the rumorspreading process in a closed system for which rumors spread over a relatively short period of time. In order to simulate the rumor-spreading process over a relatively long period of time, demographic dynamics, such as immigration and emigration rates, should be considered.

In this paper, an SIDRQ rumor propagation model is proposed to describe the dynamic model with suspicion mechanism, immune mechanism, forgetting mechanism, and guiding mechanism. Different from the existing models in a close system, our proposed model assumes that a rumor spreads in a system with constant population immigration and emigration rates and then investigates the influence of population dynamics on the rumor-spreading process.

The rest of this paper is organized as follows. An SIDRQ rumor-spreading model is established in Section 2. Section 3 deduces and calculates the basic generation number of the model. Section 4 mainly analyzes the stability of the model. Section 5 judges whether the system has controllability. In Section 6, numerical simulations are conducted followed by further discussion on the implications of these simulation results. Finally, conclusions are given in Section 7.

\section{SIDRQ Rumor-Spreading Model}

Rumors are a common form of social interaction, and the spread of rumors has an important impact on people's lives and psychology. In order to maintain people's normal life and social order, government departments will guide and control the spread of rumors to suppress the spread of rumors. In this rumor model, we divide a uniformly mixed population into five categories: I (ignorants) means people who have never heard of the rumor, $S$ (spreaders) means people who know the rumors and actively spread the dazzling people, D (doubters or skeptics) means they have heard rumors, but people who are skeptical of rumors and temporarily stop spreading rumors, $\mathrm{R}$ (removed or stilfers) means people who hear the rumors but do not spread the rumors, and Q (quarantine or control) refers to people who are unable to spread rumors because of measures taken by the government. According to Maki et al. [1], in this model, we assume that rumor spreaders only spread rumors to their own neighbors. Furthermore, we hypothesize that when the communicator is in contact with the communicator, only the initial communicator becomes immunized. The schematic diagram of the propagation process of SIDRQ rumor considering the mechanism of spreaders, ignorants, doubters, removed, and quarantine is shown in Figure 1, and its propagation rules are as follows.

(1) When ignorants contact with rumor spreaders, the ignorants become rumor-spreaders with the probability of $\lambda$, a direct immunizer with the probability of $\mu$, a skeptic with the probability of $\beta, \lambda$ is the transmission rate, $\mu$ is the rejection rate, and $\beta$ is the suspicion rate

(2) When rumor spreaders come into contact with rumorspreaders, or a suspect, or an immunized person, the initial rumor spreaders become an immunized person with a probability of $\alpha$, which is called the immunization rate

(3) When government departments take such guiding and controlling measures as title ban, speech ban, and education, rumor spreaders become leading and controlling persons with a probability of $\varepsilon$ and leading and controlling persons spontaneously become immune persons with a probability of $\gamma$, calling $\varepsilon$ the guidance rate and $\gamma$ the conversion rate

(4) Rumor spreaders become immune spontaneously with the probability of $\delta$ because they forget the rumors, and skeptics spontaneously become rumor spreaders with the probability of $\xi$, and $\delta$ is called the forgetting rate

Due to the openness and dynamicity of OSNs, we consider the variable population size of system and assume that rumors spread in the system which has the constant population immigration and emigration rates. All newly registered users enter into the ignorant class with a constant $\psi$ (i.e., immigration rate), and all existing users exit from the five classes with a constant $\rho$ (i.e., emigration rate).

A uniform network refers to a network in which the node degree values are concentrated near a certain characteristic value, such as ER random network $[11,12]$ and WS small world network [13].

Due to the introduction of the director, combined with the spreading law of SIDRQ rumors, the mean field equation of the spreading dynamics of SIDRQ rumors on a uniform network can be described as 


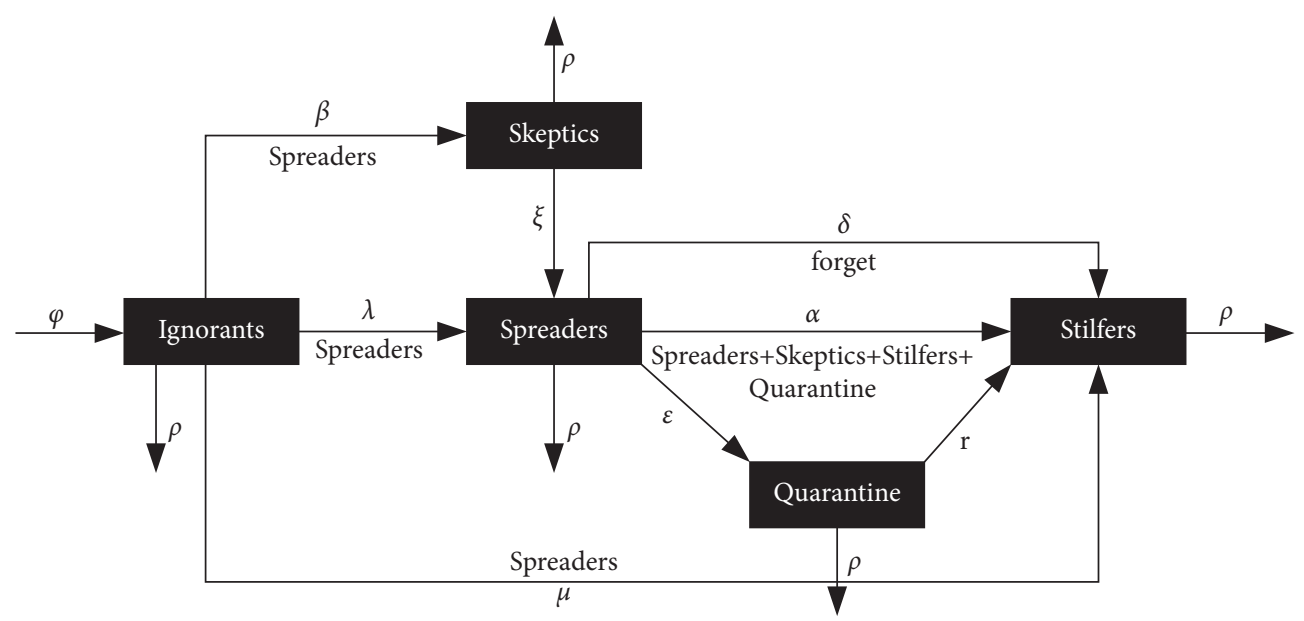

FIgURE 1: The transfer diagram of the SIDRQ model.

$$
\left\{\begin{array}{l}
\frac{\mathrm{d} S(t)}{\mathrm{d} t}=\lambda \bar{k} I(t) S(t)-\alpha \bar{k} S(t)(S(t)+D(t)+R(t)+Q(t))+\xi D(t) \\
-(\delta+\varepsilon+\rho) S(t) \\
\frac{\mathrm{d} D(t)}{\mathrm{d} t}=\beta \bar{k} I(t) S(t)-\xi D(t)-\rho D(t), \\
\frac{\mathrm{d} I(t)}{\mathrm{d} t}=\psi-(\lambda+\beta+\mu) \bar{k} I(t) S(t)-\rho I(t), \\
\frac{\mathrm{d} R(t)}{\mathrm{d} t}=\mu \bar{k} I(t) S(t)+\alpha \bar{k} S(t)(S(t)+D(t)+R(t)+Q(t)) \\
+\delta S(t)+\gamma Q(t)-\rho R(t) \\
\frac{\mathrm{d} Q(t)}{\mathrm{d} t}=\varepsilon S(t)-\gamma Q(t)-\rho Q(t)
\end{array}\right.
$$

Let $N(t)$ denote the total population of the rumorspreading system at time $t$. The number of ignorants, rumor spreaders, doubters, quarantine, and stiflers at time $t$ is denoted as, $S(t), D(t), I(t), R(t)$, and $Q(t)$. Then, we have

$$
N(t)=S(t)+D(t)+I(t)+R(t)+Q(t) .
$$

From system (1) and equation (2), we have $\frac{\mathrm{d} N(t)}{\mathrm{d} t}=\frac{\mathrm{d} S(t)}{\mathrm{d} t}+\frac{\mathrm{d} D(t)}{\mathrm{d} t}+\frac{\mathrm{d} I(t)}{\mathrm{d} t}+\frac{\mathrm{d} R(t)}{\mathrm{d} t}+\frac{\mathrm{d} Q(t)}{\mathrm{d} t}=\psi-\rho N$.

When the initial value $t=0$ in the formula, the solution is

$$
\begin{gathered}
S(0) \geq 0, \\
D(0) \geq 0, \\
I(0) \geq 0, \\
R(0) \geq 0, \\
Q(0) \geq 0 .
\end{gathered}
$$

In order to consider the nonnegativity and feasible region of the solution of SIDRQ system (1), the following conclusions can be obtained.

Theorem 1. Suppose $(S(t), D(t), I(t), R(t), Q(t))$ is the solution of system (1) under initial condition equation (4). Then, 
(i) $S(t), D(t), I(t), R(t), Q(t)$ always keep positive when $t>0$.

(ii) The feasible region $\Omega$ is a positively invariant set of system (1), which is defined as

$$
\begin{aligned}
\Omega= & (S(t), D(t), I(t), R(t), Q(t))^{T} \in R_{t}^{5}, \quad 0 \leq S(t)+D(t) \\
& \left.+I(t)+R(t)+Q(t) \leq \frac{\psi}{\rho}, \quad t>0\right\} .
\end{aligned}
$$

Proof. (i) Suppose $(S(t), D(t), I(t), R(t), Q(t))$ is the solution of system (1), when $t \in[0, T)$, where $T>0$. So, let

$$
F(t)=\min _{t}\{S(t), D(t), I(t), R(t), Q(t)\} .
$$

Here, we will prove that the solution of system (1) is positive, which implies that we only need to prove $F(t)>0$, for all $t \in[0, T)$. Assume that there exists $t^{*} \in(0, T)$ such that

$$
F\left(t^{*}\right)=\min _{t}\left\{S^{*}(t), D^{*}(t), I^{*}(t), R^{*}(t), Q^{*}(t)\right\}=0 .
$$

According to the initial condition equation (4), we can further assume $F(t)>0$ for all $t \in\left(0, t^{*}\right)$. Next, we discuss the above function $F(t)>0$ in five cases.

If $F\left(t^{*}\right)=I\left(t^{*}\right)$, then, from the first equation of system (1) and $S(t) \geq 0$, we can obtain

$$
\frac{\mathrm{d} I(t)}{\mathrm{d} t}>-(\lambda+\beta+\rho) \bar{k} I(t) S(t)-\rho I(t), \quad t \in\left[0, t^{*}\right) .
$$
has

Integrating both sides of equation (8) from 0 to $t^{*}$, one

$$
0=I\left(t^{*}\right)>I(0) \exp \left(-\int_{0}^{t_{1}}[(\lambda+\beta+\mu) \bar{k} S(t)+\rho] \mathrm{d} t\right) \geq 0,
$$

which leads to a contradiction. Furthermore, the other four cases can be discussed in a similar way. This shows that $(S(t), D(t), I(t), R(t), Q(t))$ is positive on the existence interval (i.e., for $t \in(0, T)$ ).

Then, we prove that the interval of existence of $(S(t), D(t), I(t), R(t), Q(t))$ is $[0, \infty)$. In fact, if the interval of existence is a finite interval $t \in(0, T)$, we know that $(S(t), D(t), I(t), R(t), Q(t))$ is unbounded on $[0, T)$.

From system (1) and equation (2), one has

$$
\frac{\mathrm{d} N(t)}{\mathrm{d} t}=\psi-\rho N(t)
$$

Integrating the above equation from 0 to $t$, we obtain

$$
N(t)=\frac{\psi}{\rho}+\left(N(0)-\frac{\psi}{\rho}\right) \exp (-\rho t)
$$

Hence, $N(t)$ is bounded on $[0, T)$, which implies that $S(t), D(t), I(t), R(t)$, and $Q(t)$ are bounded on $[0, T)$. This leads to a contraction. Therefore, we finally have that $(S(t), D(t), I(t), R(t), Q(t))$ is positive, for all $t \in(0, \infty)$.

(ii) According to the initial condition equation (4) and the result of (i), we have

$$
\lim _{t \longrightarrow \infty} \sup N(t)=\frac{\psi}{\rho},
$$

which implies that the feasible region $\Omega$ is positively invariant with respect to system (1). The proof of Theorem 1 is completed.

\section{Unique Existence of Solution and the Basic Reproduction Number}

The basic regeneration number is "the expected number of secondary cases arising from a typical infected individual in a fully susceptible population." In general, the basic regeneration number can be defined as the number of new infections produced by a typical infected individual in a DFE population.

According to the methodology of infectious diseases, our aim is to explore the rumor-free equilibrium $\varphi_{0}$ and the basic reproduction number $R_{0}$ of system (1). One can check that system (1) has the rumor-free equilibrium:

$$
\varphi_{0}=\left(0,0, \frac{\psi}{\rho}, 0,0\right) \text {. }
$$

Theorem 2. Suppose ( $S(t), D(t), I(t), R(t), Q(t))$ is the solution of system (1) under initial condition (4), and this explanation is unique when $t>0$.

Proof. Let

$$
x=(S(t), D(t), I(t), R(t), Q(t)) .
$$

We can obtain the following model:

$$
\frac{\mathrm{d} x}{\mathrm{~d} t}=\mathscr{F}(x)-\mathscr{V}(x)=f(x),
$$

where $\mathscr{V}=\mathscr{V}^{-}-\mathscr{V}^{+}$,

$\mathscr{F}(x)=\left(\begin{array}{c}\lambda \bar{k} I(t) S(t) \\ 0 \\ 0 \\ 0 \\ 0\end{array}\right)$,

$$
\mathscr{V}(x)=\left(\begin{array}{c}
\alpha \bar{k} S(t) \Gamma+(\delta+\varepsilon+\rho) S(t)-\xi D(t) \\
\xi D(t)+\rho D(t)-\beta \bar{k} I(t) S(t) \\
(\lambda+\beta+\mu) \bar{k} I(t) S(t)+\rho I(t)-\psi \\
\rho R(t)-\mu \bar{k} I(t) S(t)-\alpha \bar{k} S(t) \Gamma-\delta S(t)-\gamma Q(t) \\
\gamma Q(t)+\rho Q(t)-\varepsilon S(t)
\end{array}\right) .
$$

We write $\Gamma$ for the value of $S(t)+D(t)+R(t)+Q(t)$. 
Consider the $f(x)$ rumor spread of equation (15), when $x \geq 0$, then $\mathscr{F}, \mathscr{V}^{-}, \mathscr{V}^{+} \geq 0$, and when $x=0$, then $\mathscr{V}^{-}=0$. So, if $x=0$, we have $f(x) \geq 0$ and the nonnegative cone $(x \geq 0)$ is forward invariant. By Theorems 1.1.8 and 1.1.9 of [14], for each nonnegative initial condition there is a unique, nonnegative solution. The proof of Theorem 2 is completed.

The Jacobian matrices of $\mathscr{F}(x)$ and $\mathscr{V}(x)$ at equation (13) are written as

$$
\begin{aligned}
& D \mathscr{F}\left(\varphi_{0}\right)=\left(\begin{array}{ll}
F & 0 \\
0 & 0
\end{array}\right), \\
& D \mathscr{V}\left(\varphi_{0}\right)=\left(\begin{array}{ll}
V & 0 \\
J_{1} & J_{2}
\end{array}\right),
\end{aligned}
$$

where

$$
\begin{aligned}
F & =\left(\begin{array}{cc}
\lambda \bar{k} \frac{\psi}{\rho} & 0 \\
0 & 0
\end{array}\right), \\
V & =\left(\begin{array}{cc}
(\delta+\varepsilon+\rho) & -\xi \\
-\beta \bar{k} \frac{\psi}{\rho} & \xi+\rho
\end{array}\right) .
\end{aligned}
$$

Obviously, $V$ is a nonsingular matrix and $F$ is a nonnegative matrix. According to the basic reproduction number concept of $R_{0}$ given in [15],

$$
\begin{aligned}
R_{0}=\rho\left(F V^{-1}\right) & =\frac{(\xi+\rho) \lambda \bar{k}(\psi / \rho)}{(\delta+\varepsilon+\rho)(\xi+\rho)-(\xi \beta \bar{k} \psi / \rho)} \\
& =\frac{(\xi+\rho) \lambda \bar{k} \psi}{(\delta+\varepsilon+\rho)(\xi+\rho) \rho-\xi \beta \bar{k} \psi},
\end{aligned}
$$

where $\rho\left(F V^{-1}\right)$ is the spectral radius of the matrix $F V^{-1}$. $R_{0}$ :

Then, the number of equilibriums can be determined by

(a) If $R_{0}<1, \varphi_{0}$ is the unique equilibrium in $\Omega$.

(b) If $R_{0}>1$, the rumor-demise equilibrium $\varphi_{0}$ of system (1) is unstable.

Remark 1. Decomposition of $f$ leads to alternative thresholds. Therefore, we choose different $\mathscr{F}$ to calculate the basic number $R_{0}$. In the same way, when

$$
\begin{aligned}
\mathscr{F}(x)= & \left(\begin{array}{c}
\lambda \bar{k} I(t) S(t) \\
\beta \bar{k} I(t) S(t) \\
\mu \bar{k} I(t) S(t) \\
0 \\
0
\end{array}\right), \\
\mathscr{V}(x)= & \left(\begin{array}{c}
\alpha \bar{k} S(t) \Gamma+(\delta+\varepsilon+\rho) S(t)-\xi D(t) \\
\xi D(t)+\rho D(t) \\
\rho R(t)-\alpha \bar{k} S(t) \Gamma-\delta S(t)-\gamma Q(t) \\
(\lambda+\beta+\mu) \bar{k} I(t) S(t)+\rho I(t)-\psi \\
\gamma Q(t)+\rho Q(t)-\varepsilon S(t)
\end{array}\right) .
\end{aligned}
$$

From this,

$$
\begin{aligned}
& F=\left(\begin{array}{ccc}
\lambda \bar{k} \frac{\psi}{\rho} & 0 & 0 \\
\beta \bar{k} \frac{\psi}{\rho} & 0 & 0 \\
\mu \bar{k} \frac{\psi}{\rho} & 0 & 0
\end{array}\right), \\
& V=\left(\begin{array}{ccc}
\delta+\varepsilon+\rho & -\xi & 0 \\
0 & \xi+\rho & 0 \\
-\delta & 0 & \rho
\end{array}\right) .
\end{aligned}
$$

Consequently,

$$
R_{0}=\rho\left(F V^{-1}\right)=\frac{\lambda \bar{k} \psi(\rho+\xi)+\beta \bar{k} \psi \xi}{\rho(\xi+\rho)(\delta+\varepsilon+\rho)},
$$

where $\rho\left(F V^{-1}\right)$ is the spectral radius of the matrix $F V^{-1}$.

\section{Stability Analysis of SIDRQ Rumor-Spreading Model}

A detailed stability analysis of the SIDRQ rumor-spreading model is conducted in this section.

Theorem 3. If $R_{0}<1$, the rumor-demise equilibrium $\varphi_{0}$ of system (1) is locally asymptotically stable. When $R_{0}>1$, the rumor-demise equilibrium $\varphi_{0}$ of system (1) is unstable.

Proof. The Jacobian matrix of system (1) at $\varphi_{0}=(0,0,(\psi / \rho), 0,0)$ is 


$$
J\left(\varphi_{0}\right)=\left(\begin{array}{ccccc}
\lambda \bar{k} \frac{\psi}{\rho}-(\delta+\varepsilon+\rho) & \xi & 0 & 0 & 0 \\
\beta \bar{k} \frac{\psi}{\rho} & -\xi-\rho & 0 & 0 & 0 \\
-(\lambda+\beta+\mu) \bar{k} \frac{\psi}{\rho} & 0 & -\rho & 0 & 0 \\
\mu \bar{k} \frac{\psi}{\rho}+\delta & 0 & 0 & -\rho & \gamma \\
\varepsilon & 0 & 0 & 0 & -\gamma-\rho
\end{array}\right) .
$$

Computing the eigenvalues of equation (24) and applying the formula to $\left|J\left(\varphi_{0}\right)-x E\right|=0$, we have

$$
\left(\begin{array}{ccccc}
\lambda \bar{k} \frac{\psi}{\rho}-(\delta+\varepsilon+\rho)-x & \xi & 0 & 0 & 0 \\
\beta \bar{k} \frac{\psi}{\rho} & -\xi-\rho-x & 0 & 0 & 0 \\
-(\lambda+\beta+\mu) \bar{k} \frac{\psi}{\rho} & 0 & -\rho-x & 0 & 0 \\
\mu \bar{k} \frac{\psi}{\rho}+\delta & 0 & 0 & -\rho-x & \gamma \\
\varepsilon & 0 & 0 & 0 & -\gamma-\rho-x
\end{array}\right)=0 .
$$

Replacing equation (25) by equation (26),

$$
(-\gamma-\rho-x)\left|\begin{array}{cccc}
\lambda \bar{k} \frac{\psi}{\rho}-(\delta+\varepsilon+\rho)-x & \xi & 0 & 0 \\
\beta \bar{k} \frac{\psi}{\rho} & -\xi-p-x & 0 & 0 \\
-(\lambda+\beta+\mu) \bar{k} \frac{\psi}{p} & 0 & -\rho-x & 0 \\
\mu \bar{k} \frac{\psi}{\rho}+\delta & 0 & 0 & -\rho-x
\end{array}\right|=0 .
$$

We can rewrite equation (26) as 


$$
(-\gamma-\rho-x)(-\rho-x)(-\rho-x)\left|\begin{array}{cc}
\lambda \bar{k} \frac{\psi}{\rho}-(\delta+\varepsilon+\rho)-x & \xi \\
\beta \bar{k} \frac{\psi}{\rho} & -\xi-\rho-x
\end{array}\right|=0
$$

For this reason, eigenvalues of equation (24) are $x_{1}=x_{2}=-\rho, x_{3}=-\gamma-\rho, x_{4}$, and $x_{5}$ are the solutions of

$$
\left|\begin{array}{cc}
\lambda \bar{k} \frac{\psi}{\rho}-(\delta+\varepsilon+\rho)-x & \xi \\
\beta \bar{k} \frac{\psi}{\rho} & -\xi-\rho-x
\end{array}\right|=0 .
$$

We conclude from equation (28) that the solution is

$$
\left(\lambda \bar{k} \frac{\psi}{\rho}-(\delta+\varepsilon+\rho)-x\right)(-\xi-\rho-x)-\xi \beta \bar{k} \frac{\psi}{\rho}=0
$$

hence that

$$
x^{2}+\left(\xi+\rho+\delta+\varepsilon+\rho-\lambda \bar{k} \frac{\psi}{\rho}\right) x-\xi \beta \bar{k} \frac{\psi}{\rho}-\left(\lambda \bar{k} \frac{\psi}{\rho}-(\delta+\varepsilon+\rho)\right)(\rho+\xi) \text {. }
$$

According to the assumption that $R_{0}<1$, we have

$$
\begin{gathered}
-\xi \beta \bar{k} \frac{\psi}{\rho}-\left(\lambda \bar{k} \frac{\psi}{\rho}-(\delta+\varepsilon+\rho)\right)(\rho+\xi)>0, \\
(\xi+\rho) \lambda \bar{k} \psi<(\delta+\varepsilon+\rho)(\xi+\rho) \rho-\xi \beta \bar{k} \psi .
\end{gathered}
$$

Applying equation (32),

$$
(\xi+\rho) \lambda \bar{k} \psi<(\delta+\varepsilon+\rho)(\xi+\rho) \rho .
$$

Thus,

$$
\lambda \bar{k} \frac{\psi}{\rho}<\delta+\varepsilon+\rho
$$

and finally,

$$
\left(\xi+\rho+\delta+\varepsilon+\rho-\lambda \bar{k} \frac{\psi}{\rho}\right)>0 .
$$

We conclude from equations (31) and (35) that $x_{4}<0$ and $x_{5}<0$; hence, based on the Routh-Hurwitz criterion
[16], the rumor-demise equilibrium $\varphi_{0}$ of system (1) is locally asymptotically stable if $R_{0}<1$, and finally, it is unstable when $R_{0}>1$. From this, we complete the proof of Theorem 3.

Theorem 4. If $R_{0}<1$, the rumor-demise equilibrium $\varphi_{0}$ of system (1) is globally asymptotically stable. When $R_{0}>1$, the rumor-demise equilibrium $\varphi_{0}$ of system (1) is unstable.

Proof. Via the constructive Lyapunov function,

$$
V(t)=\frac{1}{\delta+\varepsilon+\rho} S(t)+\frac{\xi}{(\xi+\rho)(\delta+\varepsilon+\rho)} D(t)
$$

and taking derivative to the both sides of the equality gives

$$
\dot{V}(t)=\frac{1}{\delta+\varepsilon+\rho} \dot{S}(t)+\frac{\xi}{(\xi+\rho)(\delta+\varepsilon+\rho)} \dot{D}(t)
$$

Combining $\dot{S}(t)$ with $\dot{D}(t)$, we can rewrite (37) as

$$
\begin{aligned}
\dot{V}(t)= & \frac{\rho+\varepsilon}{(\delta+\varepsilon+\rho)(\rho+\varepsilon)}(\lambda \bar{k} I(t) S(t)-\alpha \bar{k} S(t) \Gamma+\xi D(t) \\
& -(\delta+\varepsilon+\rho) S(t))+\frac{\xi}{(\delta+\varepsilon+\rho)(\rho+\varepsilon)}(\beta \bar{k} I(t) S(t)-\xi D(t)-\rho D(t)) \\
= & \frac{1}{(\delta+\varepsilon+\rho)(\rho+\varepsilon)}((\rho+\varepsilon) \lambda \bar{k} I(t) S(t)+\xi \beta \bar{k} I(t) S(t)) \\
& -S(t)-\frac{\alpha \bar{k} S(t) \Gamma}{\delta+\varepsilon+\rho}
\end{aligned}
$$




$$
\begin{aligned}
& \leq \frac{(\rho+\varepsilon) \lambda \bar{k} I(t) S(t)+\xi \beta \bar{k} I(t) S(t)}{(\delta+\varepsilon+\rho)(\rho+\varepsilon)}-S(t) \\
& =\left(\frac{(\rho+\varepsilon) \lambda \bar{k} I(t)+\xi \beta \bar{k} I(t)}{(\delta+\varepsilon+\rho)(\rho+\varepsilon)}-1\right) S(t) .
\end{aligned}
$$

Applying equations (12) and (13), we obtain $I(t) \leq(\psi / \rho)$, and we can rewrite equation (38) as

$$
\dot{V}(t) \leq\left(\frac{(\rho+\varepsilon) \lambda \bar{k} \psi+\xi \beta \bar{k} \psi}{\rho(\delta+\varepsilon+\rho)(\rho+\varepsilon)}-1\right) S(t)
$$

In the same way, there exists

$$
\dot{V}(t) \leq\left(R_{0}-1\right) S(t) .
$$

Suppose, for the moment $R_{0}<1$, we see that $\dot{V}(t)<0$. Set $S(t)=0$; let us compute $\dot{V}(t)=0$; hence, the rumor-demise equilibrium $\varphi_{0}$ of system (1) is globally asymptotically stable.
Next, we will prove the uniqueness of the rumorprevailing equilibrium $\varphi^{*}$.

Theorem 5. If $R_{0}>1$, system (41) has a unique positive solution of $\varphi^{*}=\left(S^{*}, D^{*}, I^{*}, R^{*}, Q^{*}\right)$.

Proof. Assuming that $\varphi^{*}=\left(S^{*}, D^{*}, I^{*}, R^{*}, Q^{*}\right)$ is an equilibrium of system (1), we can obtain

$$
\left\{\begin{array}{l}
\lambda \bar{k} I(t) S(t)-\alpha \bar{k} S(t) \Gamma+\xi D(t)-(\delta+\epsilon+\rho) S(t)=0 \\
\beta \bar{k} I(t) S(t)-\xi D(t)-\rho D(t)=0 \\
\psi-(\lambda+\beta+\mu) \bar{k} I(t) S(t)-\rho I(t)=0 \\
\mu \bar{k} I(t) S(t)+\alpha \bar{k} S(t) \Gamma+\delta S(t)+\gamma Q(t)-\rho R(t)=0 \\
\epsilon S(t)-\gamma Q(t)-\rho Q(t)=0
\end{array}\right.
$$

On the basis of systems, from the 1 st, 2 nd, 4 th, and 5 th equations in the system (41), we obtain

$$
S(t)+D(t)+R(t)+Q(t)=\frac{(\lambda+\beta+\mu) \bar{k} I(t) S(t)}{\rho} .
$$

It follows from the 2 nd equation of (41) that

$$
D(t)=\frac{\beta \bar{k} I(t) S(t)}{\rho+\xi} .
$$

According to the 3 rd equation of (41),

$$
I(t)=\frac{\psi}{\rho+(\lambda+\beta+\mu) \bar{k} S(t)} .
$$

So, let us substitute equations (42)-(44) into the 1st equation of (41), and we have

$$
\begin{aligned}
& -\left((\rho+\xi) \alpha(\lambda+\beta+\mu) \bar{k}^{2} \psi+\rho(\rho+\xi)(\lambda+\beta+\mu) \bar{k}(\delta+\varepsilon+\rho)\right) S^{2}(t) \\
& +(\rho(\rho+\xi) \lambda \bar{k} \psi+\rho \xi \beta \bar{k} \psi-\rho(\rho+\xi) \rho(\delta+\varepsilon+\rho)) S(t)=0 . \\
& \quad(\rho+\xi) \lambda \bar{k} \psi+\xi \beta \bar{k} \psi>\rho(\rho+\xi)(\delta+\varepsilon+\rho) .
\end{aligned}
$$

When $R_{0}>1$, it can be concluded that 
Then,

$$
\rho(\rho+\xi) \lambda \bar{k} \psi+\rho \xi \beta \bar{k} \psi-\rho(\rho+\xi) \rho(\delta+\varepsilon+\rho)>0 .
$$

Consequently, equation (47) has a unique positive solution. In conclusion, when $R_{0}>1$, system (41) have a unique positive solution.
Theorem 6. If $R_{0}>1$, the rumor-demise equilibrium $\varphi^{*}$ of system (1) is locally asymptotically stable.

Proof. The Jacobian at $\varphi^{*}=\left(S^{*}, D^{*}, I^{*}, R^{*}, Q^{*}\right)$ is

$$
J\left(\varphi^{*}\right)=\left(\begin{array}{ccccc}
\mathscr{A} & -\alpha \bar{k} S^{*}+\xi & \lambda \bar{k} S^{*} & -\alpha \bar{k} S^{*} & -\alpha \bar{k} S^{*} \\
\beta \bar{k} I^{*} & -\xi-\rho & \beta \bar{k} S^{*} & 0 & 0 \\
-(\lambda+\beta+\mu) \bar{k} I^{*} & 0 & \mathscr{C} & 0 & 0 \\
\mathscr{B} & \alpha \bar{k} S^{*} & \mu \bar{k} S^{*} & \alpha \bar{k} S^{*}-\rho & \alpha \bar{k} S^{*}+\gamma \\
\varepsilon & 0 & 0 & 0 & -\gamma-\rho
\end{array}\right) .
$$

We will use the symbols $\mathscr{A}, \mathscr{B}$, and $\mathscr{C}$ to denote It follows from $\left|J\left(\varphi^{*}\right)-x E\right|=0$ that

$$
\begin{aligned}
& \mathscr{A}=\lambda \bar{k} I^{*}-\alpha \bar{k}\left(S^{*}+D^{*}+R^{*}+Q^{*}\right)-\alpha \bar{k} S^{*}-(\delta+\varepsilon+\rho), \\
& \mathscr{B}=\mu \bar{k} I^{*}+\alpha \bar{k}\left(S^{*}+D^{*}+R^{*}+Q^{*}\right)+\alpha \bar{k} S^{*}+\delta, \\
& \mathscr{C}=-(\lambda+\beta+\mu) \bar{k} S^{*}-\rho .
\end{aligned}
$$

$$
\left|\begin{array}{ccccc}
\mathscr{A}-x & -\alpha \bar{k} S^{*}+\xi & \alpha \bar{k} S^{*} & -\alpha \bar{k} S^{*} & -\alpha \bar{k} S^{*} \\
\beta \bar{k} I^{*} & -\xi-\rho-x & \beta \bar{k} S^{*} & 0 & 0 \\
-(\lambda+\beta+\mu) \bar{k} I^{*} & 0 & \mathscr{C}-x & 0 & 0 \\
\mathscr{B} & \alpha \bar{k} S^{*} & \mu \bar{k} S^{*} & \alpha \bar{k} S^{*}-\rho-x & \alpha \bar{k} S^{*}+\gamma \\
\varepsilon & 0 & 0 & 0 & -\gamma-\rho-x
\end{array}\right|=0 .
$$

Simplifying gives

$$
\begin{aligned}
& (-\rho-x)\left|\begin{array}{ccccc}
1 & 1 & 1 & 1 & 1 \\
\beta \bar{k} I^{*} & -\xi-\rho-x & \beta \bar{k} S^{*} & 0 & 0 \\
-(\lambda+\beta+\mu) \bar{k} I^{*} & 0 & \mathscr{C}-x & 0 & 0 \\
\mathscr{B} & \alpha \bar{k} S^{*} & \mu \bar{k} S^{*} & \alpha \bar{k} S^{*}-\rho-x & \alpha \bar{k} S^{*}+\gamma \\
\varepsilon & 0 & 0 & 0 & -\gamma-\rho-x
\end{array}\right|=0 \text {, } \\
& (-\rho-x)(-\gamma-\rho-x)\left|\begin{array}{cccc}
1 & 1 & 1 & 1 \\
\beta \bar{k} I^{*} & -\xi-\rho-x & \beta \bar{k} S^{*} & 0 \\
-(\lambda+\beta+\mu) \bar{k} I^{*} & 0 & \mathscr{C}-x & 0 \\
\mathscr{B} & \alpha \bar{k} S^{*} & \mu \bar{k} S^{*} & \alpha \bar{k} S^{*}-\rho-x
\end{array}\right|=0
\end{aligned}
$$




$$
\begin{gathered}
(-\rho-x)(-\gamma-\rho-x)\left|\begin{array}{cccc}
1 & 1 & 1 & 1 \\
\beta \bar{k} I^{*} & -\xi-\rho-x & \beta \bar{k} S^{*} & 0 \\
-(\lambda+\beta+\mu) \bar{k} I^{*} & 0 & \mathscr{C}-x & 0 \\
\mathscr{H} & \rho+x & \mathscr{D} & 0
\end{array}\right|=0, \\
(-\rho-x)(-\gamma-\rho-x)\left|\begin{array}{ccc}
\beta \bar{k} I^{*} & -\xi-\rho-x & \beta \bar{k} S^{*} \\
-(\lambda+\beta+\mu) \bar{k} I^{*} & 0 & \mathscr{C}-x \\
\mathscr{H} & \rho+x & \mathscr{D}
\end{array}\right|=0 .
\end{gathered}
$$

We write $\mathscr{D}, \mathscr{H}$ for the value of

$$
\begin{aligned}
& \mathscr{D}=\mu \bar{k} S^{*}-\alpha \bar{k} S^{*}+\rho+x, \\
& \mathscr{H}=\mu \bar{k} I^{*}+\alpha \bar{k}\left(S^{*}+D^{*}+R^{*}+Q^{*}\right)+\delta+\varepsilon+\rho+x .
\end{aligned}
$$

Combining equation (41), so we have

$$
\alpha \bar{k}\left(S^{*}+D^{*}+R^{*}+Q^{*}\right)+(\delta+\varepsilon+\rho)=\lambda \bar{k} I^{*}+\xi \frac{D^{*}}{S^{*}} .
$$

$\lambda \bar{k} I^{*} S^{*}-\alpha \bar{k} S^{*}\left(S^{*}+D^{*}+R^{*}+Q^{*}\right)+\xi D^{*}-(\delta+\varepsilon+\rho) S^{*}=0$,

$$
(-\rho-x)(-\gamma-\rho-x)\left|\begin{array}{ccc}
\beta \bar{k} I^{*} & -\xi-\rho-x & \beta \bar{k} S^{*} \\
-(\lambda+\beta+\mu) \bar{k} I^{*} & 0 & \mathscr{C}-x \\
(\mu+\lambda) \bar{k} I^{*}+\xi \frac{D^{*}}{S^{*}}+x & \rho+x & \mathscr{D}
\end{array}\right|=0,
$$

and we have

$$
(-\rho-x)(-\gamma-\rho-x)\left|\begin{array}{ccc}
\beta \bar{k} I^{*} & -\xi-\rho-x & \beta \bar{k} S^{*} \\
-(\lambda+\beta+\mu) \bar{k} I^{*} & 0 & \mathscr{C}-x \\
\xi \frac{D^{*}}{S^{*}}+x & -\xi & -\lambda \bar{k} S^{*}-\alpha \bar{k} S^{*}
\end{array}\right|=0 .
$$

From this, we conclude from equation (61) that $x_{1}=-\rho$ and $x_{2}=-\gamma-\rho$, and $x_{3}, x_{4}$, and $x_{5}$ are solutions of equation (62):

$$
\left|\begin{array}{ccc}
\beta \bar{k} I^{*} & -\xi-\rho-x & \beta \bar{k} S^{*} \\
-(\lambda+\beta+\mu) \bar{k} I^{*} & 0 & \mathscr{C}-x \\
\xi \frac{D^{*}}{S^{*}}+x & -\xi & -\lambda \bar{k} S^{*}-\alpha \bar{k} S^{*}
\end{array}\right|=0
$$


It follows that

$$
\begin{aligned}
x^{3} & +\left(\xi \frac{D^{*}}{S^{*}}+\rho+\xi+(\lambda+\beta+\mu) \bar{k} S^{*}+\rho\right) x^{2}+\left(\xi \frac{D^{*}}{S^{*}}(\rho+\xi)\right. \\
& +\xi \frac{D^{*}}{S^{*}}\left((\lambda+\beta+\mu) \bar{k} S^{*}+\rho\right)+(\rho+\xi)\left((\lambda+\beta+\mu) \bar{k} S^{*}+\rho\right) \\
& \left.+(\lambda+\beta+\mu) \bar{k} I^{*}\left(\alpha \bar{k} S^{*}+\lambda \bar{k} S^{*}\right)-\beta \bar{k} I^{*} \xi\right) x+\left(\xi \frac{D^{*}}{S^{*}}(\rho+\xi)\right. \\
& \times\left((\lambda+\beta+\mu) \bar{k} S^{*}+\rho\right)+\xi \beta \bar{k} S^{*}(\lambda+\beta+\mu) \bar{k} I^{*}+(\lambda+\beta+\mu) \\
& \left.\times \bar{k} I^{*}(\xi+\rho)\left(\lambda \bar{k} S^{*}+\alpha \bar{k} S^{*}\right)-\beta \bar{k} I^{*} \xi\left((\lambda+\beta+\mu) \bar{k} S^{*}+\rho\right)\right)=0 .
\end{aligned}
$$

Set

$$
\begin{aligned}
a_{3}= & 1, \\
a_{2}= & \xi \frac{D^{*}}{S^{*}}+\rho+\xi+(\lambda+\beta+\mu) \bar{k} S^{*}+\rho, \\
a_{1}= & \xi \frac{D^{*}}{S^{*}}(\rho+\xi)+\xi \frac{D^{*}}{S^{*}}\left((\lambda+\beta+\mu) \bar{k} S^{*}+\rho\right)+(\rho+\xi)((\lambda+\beta \\
& \left.+\mu) \bar{k} S^{*}+\rho\right)+(\lambda+\beta+\mu) \bar{k} I^{*}\left(\alpha \bar{k} S^{*}+\lambda \bar{k} S^{*}\right)-\beta \bar{k} I^{*} \xi, \\
a_{0}= & \xi \frac{D^{*}}{S^{*}}(\rho+\xi)\left((\lambda+\beta+\mu) \bar{k} S^{*}+\rho\right)+\xi \beta \bar{k} S^{*}(\lambda+\beta+\mu) \bar{k} I^{*}+(\lambda+\beta \\
& +\mu) \bar{k} I^{*}(\xi+\rho)\left(\lambda \bar{k} S^{*}+\alpha \bar{k} S^{*}\right)-\beta \bar{k} I^{*} \xi\left((\lambda+\beta+\mu) \bar{k} S^{*}+\rho\right) .
\end{aligned}
$$

According to Lienard and Chipart theorem, order for equation (63) has three negative real roots which are $x_{3}, x_{4}$, and $x_{5}$. Equation (63) must satisfy $a_{0}>0, a_{1}>0, a_{2}>0$, $a_{3}>0$, and $a_{1} a_{2}>a_{0} a_{3}$.

Obviously, $a_{2}>0$ and $a_{3}>0$. The proof will be divided into three steps: $a_{0}>0, a_{1}>0$, and $a_{1} a_{2}>a_{0} a_{3}$.

(i) Let us firstly prove that $a_{1}>0$ by

$$
D^{*}=\frac{\beta \bar{k} I^{*} S^{*}}{\rho+\xi} \text {. }
$$

Equation (65) then can be transformed into

$$
\xi \frac{D^{*}}{S^{*}}(\rho+\xi)\left((\lambda+\beta+\mu) \bar{k} S^{*}+\rho\right)-\beta \bar{k} \xi I^{*}\left((\lambda+\beta+\mu) \bar{k} S^{*}+\rho\right)=0 .
$$

It follows that

$$
a_{0}=\xi \beta \bar{k} S^{*}(\lambda+\beta+\mu) \bar{k} I^{*}+(\lambda+\beta+\mu) \bar{k} I^{*}(\xi+\rho)\left(\lambda \bar{k} S^{*}+\alpha \bar{k} S^{*}\right)>0 \text {. }
$$


(iii) According to equation (64), we have

$$
\begin{aligned}
& a_{1} a_{2}-a_{0} a_{3}=\left[\xi \frac{D^{*}}{S^{*}}\left((\lambda+\beta+\mu) \bar{k} S^{*}+\rho\right)+(\rho+\xi)\left((\lambda+\beta+\mu) \bar{k} S^{*}+\rho\right)\right. \\
&\left.+(\lambda+\beta+\mu) \bar{k} I^{*}\left(\alpha \bar{k} S^{*}+\lambda \bar{k} S^{*}\right)\right]\left(\xi \frac{D^{*}}{S^{*}}+\rho+\xi\right. \\
&\left.+(\lambda+\beta+\mu) \bar{k} S^{*}+\rho\right)-\xi \beta \bar{k} S^{*}(\lambda+\beta+\mu) \bar{k} I^{*} \\
&-(\lambda+\beta+\mu) \bar{k} I^{*}(\xi+\rho)\left(\lambda \bar{k} S^{*}+\alpha \bar{k} S^{*}\right) .
\end{aligned}
$$

It is easy to show that

$$
\begin{aligned}
a_{1} a_{2}-a_{0} a_{3}= & \left(\xi \frac{D^{*}}{S^{*}}+(\lambda+\beta+\mu) \bar{k} S^{*}+\rho\right)\left(\xi \frac{D^{*}}{S^{*}}\left((\lambda+\beta+\mu) \bar{k} S^{*}+\rho\right)\right. \\
& +(\rho+\xi)\left((\lambda+\beta+\mu) \bar{k} S^{*}+\rho\right)+(\lambda+\beta+\mu) \bar{k} I^{*}\left(\lambda \bar{k} S^{*}\right. \\
& \left.\left.+\alpha \bar{k} S^{*}\right)\right)+(\rho+\xi)\left(\xi \frac{D^{*}}{S^{*}} \rho+(\rho+\xi)\left((\lambda+\beta+\mu) \bar{k} S^{*}+\rho\right)\right)>0 .
\end{aligned}
$$

As mentioned, according to equations (67), (69), and (71), equation (63) has three negative real roots and contented Lienard and Chipart theorem. Therefore, when $R_{0}>1$, the rumor-demise equilibrium $\varphi^{*}$ of system (1) is locally asymptotically stable.

Remark 2. In the foregoing, when $R_{0}>1$, it can be concluded that the system is locally asymptotically stable.
However, our research shows that not all rumor-spreading models are stable at $R_{0}>1$, and we will give you a practical example. Add a nonlinear term to system (1), i.e., when a doubter contacts a rumor spreader, the doubter becomes a rumor spreader with probability $\eta$. Propagation process is as shown in Figure 2.

The system can be described as

$$
\left\{\begin{array}{l}
\frac{\mathrm{d} S(t)}{\mathrm{d} t}=\lambda \bar{k} I(t) S(t)-\alpha \bar{k} S(t)(S(t)+D(t)+R(t)+Q(t))+\xi D(t) \\
+\eta \bar{k} S(t) D(t)-(\delta+\varepsilon+\rho) S(t), \\
\frac{\mathrm{d} D(t)}{\mathrm{d} t}=\beta \bar{k} I(t) S(t)-\eta \bar{k} S(t) D(t)-\xi D(t)-\rho D(t), \\
\frac{\mathrm{d} I(t)}{\mathrm{d} t}=\psi-(\lambda+\beta+\mu) \bar{k} I(t) S(t)-\rho I(t), \\
\frac{\mathrm{d} R(t)}{\mathrm{d} t}=\mu \bar{k} I(t) S(t)+\alpha \bar{k} S(t)(S(t)+D(t)+R(t)+Q(t)) \\
+\delta S(t)+\gamma Q(t)-\rho R(t), \\
\frac{\mathrm{d} Q(t)}{\mathrm{d} t}=\varepsilon S(t)-\gamma Q(t)-\rho Q(t) .
\end{array}\right.
$$




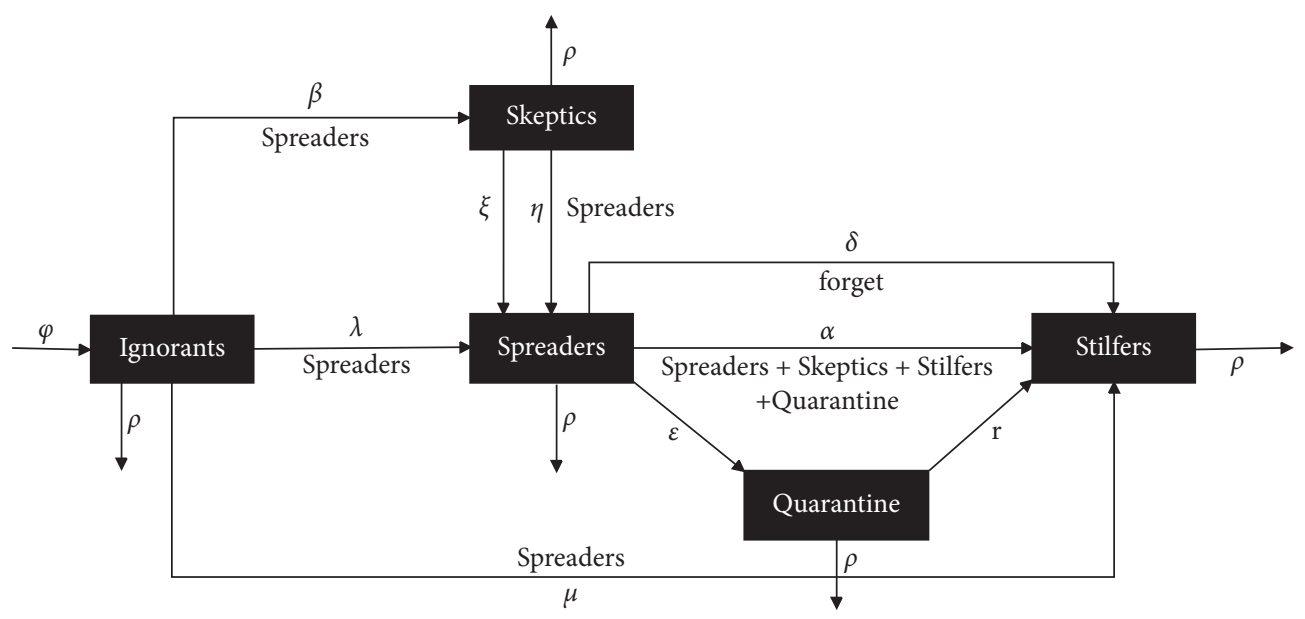

FIGURE 2: The transfer diagram of the SIDRQ model when adding a nonlinear term.

Although system (72) is locally asymptotically stable at $\varphi_{0}$, when $R_{0}<1$, it is not necessarily asymptotically stable at $\varphi^{*}$.
In the same way, the proof of Theorem 3 is similar to that of Theorem 6.

Proof. When $R_{0}>1$,

$$
\left\{\begin{array}{l}
\lambda \bar{k} I(t) S(t)-\alpha \bar{k} S(t)(S(t)+D(t)+R(t)+Q(t))+\xi D(t) \\
+\eta \bar{k} S(t) D(t)-(\delta+\varepsilon+\rho) S(t)=0, \\
\beta \bar{k} I(t) S(t)-\eta \bar{k} S(t) D(t)-\xi D(t)-\rho D(t)=0, \\
\psi-(\lambda+\beta+\mu) \bar{k} I(t) S(t)-\rho I(t)=0, \\
\mu \bar{k} I(t) S(t)+\alpha \bar{k} S(t)(S(t)+D(t)+R(t)+Q(t)) \\
+\delta S(t)+\gamma Q(t)-\rho R(t)=0, \\
\varepsilon S(t)-\gamma Q(t)-\rho Q(t)=0 .
\end{array}\right.
$$

We can say that equation (73) has a unique solution of $\varphi^{*}=\left(S^{*}, D^{*}, I^{*}, R^{*}, Q^{*}\right)$.
It is obvious that computing the Jacobian matrix $J\left(\varphi^{*}\right)$ of equation (73) has

$$
J\left(\varphi^{*}\right)=\left(\begin{array}{ccccc}
A & -\alpha \bar{k} S^{*}+\xi+\eta \bar{k} S^{*} & \lambda \bar{k} S^{*} & -\alpha \bar{k} S^{*} & -\alpha \bar{k} S^{*} \\
\beta \bar{k} I^{*}-\eta \bar{k} D^{*} & -\xi-\eta \bar{k} S^{*}-\rho & \beta \bar{k} S^{*} & 0 & 0 \\
-(\lambda+\beta+\mu) \bar{k} I^{*} & 0 & C & 0 & 0 \\
B & \alpha \bar{k} S^{*} & \mu \bar{k} S^{*} & \alpha \bar{k} S^{*}-\rho & \alpha \bar{k} S^{*}+\gamma \\
\varepsilon & 0 & 0 & 0 & -\gamma-\rho
\end{array}\right) .
$$

We will use the letters $A, B$, and $C$ to denote

$$
\begin{aligned}
& A=\lambda \bar{k} I^{*}-\alpha \bar{k}\left(S^{*}+D^{*}+R^{*}+Q^{*}\right)-\alpha \bar{k} S^{*}+\eta \bar{k} D^{*}-(\delta+\varepsilon+\rho), \\
& B=\mu \bar{k} I^{*}+\alpha \bar{k}\left(S^{*}+D^{*}+R^{*}+Q^{*}\right)+\alpha \bar{k} S^{*}+\delta, \\
& C=-(\lambda+\beta+\mu) \bar{k} S^{*}-\rho .
\end{aligned}
$$


We calculate from $\left|J\left(\varphi^{*}\right)-x E\right|=0$ that the eigenvalue is as follows:

$$
\left|\begin{array}{ccccc}
A-x & -\alpha \bar{k} S^{*}+\eta \bar{k} S^{*}+\xi & \lambda \bar{k} S^{*} & -\alpha \bar{k} S^{*} & -\alpha \bar{k} S^{*} \\
\beta \bar{k} I^{*}-\eta \bar{k} D^{*} & -\xi-\eta \bar{k} S^{*}-\rho-x & \beta \bar{k} S^{*} & 0 & 0 \\
-(\lambda+\beta+\mu) \bar{k} I^{*} & 0 & C-x & 0 & 0 \\
B & \alpha \bar{k} S^{*} & \mu \bar{k} S^{*} & \alpha \bar{k} S^{*}-\rho-x & \alpha \bar{k} S^{*}+\gamma \\
\varepsilon & 0 & 0 & 0 & -\gamma-\rho-x
\end{array}\right|=0
$$

and it is apparent that

$$
(-\rho-x)(-\rho-\gamma-x)\left|\begin{array}{ccc}
\xi \frac{D^{*}}{S^{*}}+x & -\xi-\eta \bar{k} S^{*} & -\lambda \bar{k} S^{*}-\alpha \bar{k} S^{*} \\
\eta \bar{k} D^{*}-\beta \bar{k} I^{*} & \xi+\eta \bar{k} S^{*}+\rho+x & \beta \bar{k} S^{*} \\
(\lambda+\beta+\mu) \bar{k} I^{*} & 0 & (\lambda+\beta+\mu) \bar{k} S^{*}+\rho+x
\end{array}\right|=0
$$

Hence, we have thus proved $x_{1}=-\rho$ and $x_{2}=-\rho-\gamma$, and $x_{3}, x_{4}$, and $x_{5}$ are eigenvalues of $f(x)=a_{3} x^{3}+a_{2} x^{2}+a_{1} x+a_{0}$. Therein,

$$
\begin{aligned}
a_{3}= & 1 \\
a_{2}= & \xi \frac{D^{*}}{S^{*}}+\rho+\xi+\eta \bar{k} S^{*}+(\lambda+\beta+\mu) \bar{k} S^{*}+\rho \\
a_{1}= & \xi \frac{D^{*}}{S^{*}}\left(\rho+\xi+\eta \bar{k} S^{*}\right)+\xi \frac{D^{*}}{S^{*}}\left((\lambda+\beta+\mu) \bar{k} S^{*}+\rho\right) \\
& +\left(\rho+\xi+\eta \bar{k} S^{*}\right)\left((\lambda+\beta+\mu) \bar{k} S^{*}+\rho\right) \\
& +(\lambda+\beta+\mu) \bar{k} I^{*}\left(\alpha \bar{k} S^{*}+\lambda \bar{k} S^{*}\right)+\left(\xi+\eta \bar{k} S^{*}\right)\left(\eta \bar{k} D^{*}-\beta \bar{k} I^{*}\right), \\
a_{0}= & \xi \frac{D^{*}}{S^{*}}\left(\rho+\xi+\eta \bar{k} S^{*}\right)\left((\lambda+\beta+\mu) \bar{k} S^{*}+\rho\right) \\
& +\left(\xi+\eta \bar{k} S^{*}\right) \beta \bar{k} S^{*}(\lambda+\beta+\mu) \bar{k} I^{*} \\
& +(\lambda+\beta+\mu) \bar{k} I^{*}\left(\xi+\rho+\eta \bar{k} S^{*}\right)\left(\lambda \bar{k} S^{*}+\alpha \bar{k} S^{*}\right) \\
& +\left(\xi+\eta \bar{k} S^{*}\right)\left(\eta \bar{k} D^{*}-\beta \bar{k} I^{*}\right)\left((\lambda+\beta+\mu) \bar{k} S^{*}+\rho\right) .
\end{aligned}
$$

According to our condition $R_{0}>1$, we cannot get $a_{1}, a_{0}>0$ and $a_{1} a_{2}>a_{0} a_{3}$. So, the system is not necessarily asymptotically stable. 


\section{Controllability Analysis of the SIDRQ Rumor-Spreading Model}

Controllability is an important concept in modern control theory. Controllability research helps us to understand complex systems more deeply. The rumor propagation system is a typical complex nonlinear system, and the controllability analysis of the nonlinear system can be understood as the feasibility study of changing rumor propagation from one dynamic behavior state to another dynamic behavior through appropriate control strategy, which provides the certain reference value for rumor control.

The dynamic system of SIDRQ spreading of rumors is

$$
\left\{\begin{array}{l}
\frac{\mathrm{d} S(t)}{\mathrm{d} t}=\lambda \bar{k} I(t) S(t)-\alpha \bar{k} S(t)(S(t)+D(t)+R(t)+Q(t))+\xi D(t) \\
-(\delta+\varepsilon) S(t)-\rho(t) S(t) \\
\frac{\mathrm{d} D(t)}{\mathrm{d} t}=\beta \bar{k} I(t) S(t)-\xi D(t)-\rho(t) D(t) \\
\frac{\mathrm{d} I(t)}{\mathrm{d} t}=\psi-(\lambda+\beta+\mu) \bar{k} I(t) S(t)-\rho(t) I(t) \\
\frac{\mathrm{d} R(t)}{\mathrm{d} t}=\mu \bar{k} I(t) S(t)+\alpha \bar{k} S(t)(S(t)+D(t)+R(t)+Q(t)) \\
+\delta S(t)+\gamma Q(t)-\rho(t) R(t) \\
\frac{\mathrm{d} Q(t)}{\mathrm{d} t}=\varepsilon S(t)-\gamma Q(t)-\rho(t) Q(t)
\end{array}\right.
$$

where $\rho(t)$ is the control variable.

Theorem 7. System (79) is completely controllable.

Proof. We can write SIDRQ spreading of rumors system (79) as

$$
\dot{x}=f(x, t)+G(t) x u(t),
$$

where

$$
\begin{array}{r}
x(t)=(S(t), D(t), I(t), R(t), Q(t))^{T}, \\
u(t)=\rho(t) .
\end{array}
$$

Hence,

$$
\begin{aligned}
F(x, t) & =\left(\begin{array}{c}
\lambda \bar{k} I(t) S(t)-\alpha \bar{k} S(t) \Gamma+\xi D(t)-(\delta+\varepsilon) S(t) \\
\beta \bar{k} I(t) S(t)-\xi D(t) \\
\psi-(\lambda+\beta+\mu) \bar{k} I(t) S(t) \\
\mu \bar{k} I(t) S(t)+\alpha \bar{k} S(t) \Gamma+\delta S(t)+\gamma Q(t) \\
\varepsilon S(t)-\gamma Q(t)
\end{array}\right), \\
G(t) & =\left(\begin{array}{ccccc}
-1 & 0 & 0 & 0 & 0 \\
0 & -1 & 0 & 0 & 0 \\
0 & 0 & -1 & 0 & 0 \\
0 & 0 & 0 & -1 & 0 \\
0 & 0 & 0 & 0 & -1
\end{array}\right) .
\end{aligned}
$$

It is found that if the $G(t)$ matrices satisfy certain conditions, the system is completely controllable for all intervals $[0, T]$ regardless of the form of $f(x, t)$.
Construct

$$
V(x, t)=x(t)^{T} P(x) x(t),
$$




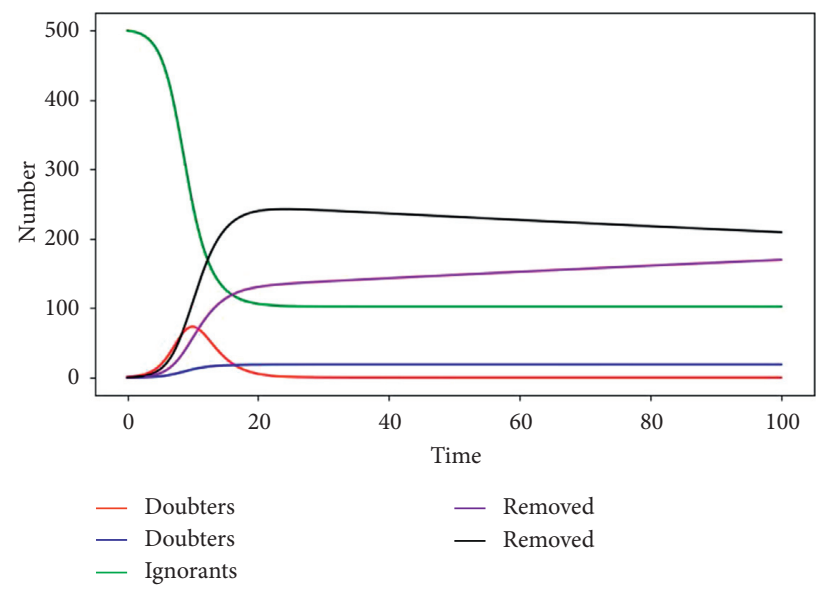

Figure 3: The stability of $\varphi_{0}$ in system (1) with $\mathrm{R}_{0}<1$.

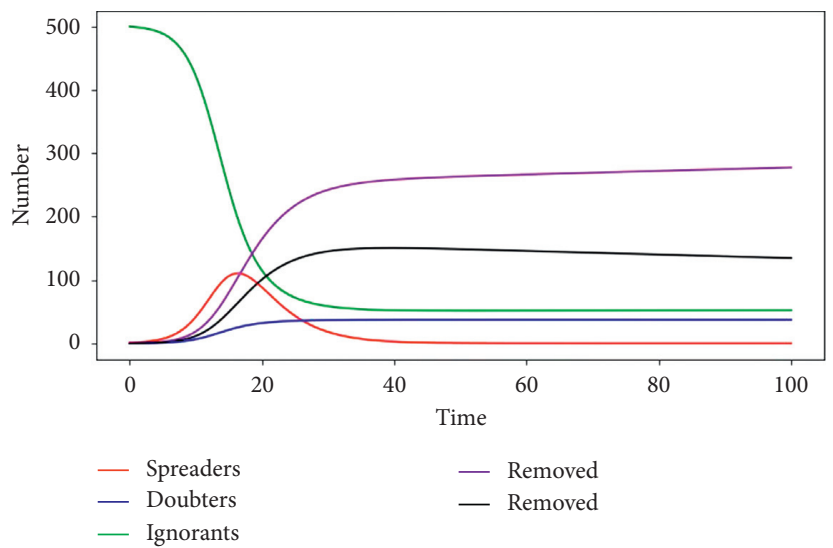

Figure 4: The stability of $\varphi^{*}$ in system (1) with $\mathrm{R}_{0}>1$.

where $P(x)$ is any positive definite matrix. Then,

$$
\dot{V}(x, t)=2 x^{T} P(x) f+x^{T}\left(P G+G^{T} P\right) x u+x^{T} \dot{P} x .
$$

We know from equation (83) that $P G+G^{T} P$ is negative definite. Therefore, according to [17], system (79) is completely controllable. Proof is done.

\section{Numerical Simulations}

This section mainly studies the propagation dynamic properties of the SIDRQ rumor-spreading model on a uniform network through simulation experiments.

In this section, numerical simulation is carried out to verify the analysis results in Section 4 . Section 3 conducts a detailed stability analysis of the SIDRQ rumor propagation model. In our analysis, we find that the threshold parameter $R_{0}$ is closely related to the dynamic characteristics of the model. When $R_{0}<1, \varphi_{0}$ is globally asymptotically stable, and when $R_{0}>1, \varphi^{*}$ is locally asymptotically stable in the feasible region. We assume that the total number of people at time $t=0$ is 501 , and there is only one spreader in the system, and the rest are ignorants, i.e., $N(0)=100001, S(0)=1$,
$I(0)=100000, D(0)=0, R(0)=0$, and $Q(0)=0$. The results of numerical simulations are as shown in Figures 3 and 4 which validate the theoretical analysis presented in Theorems 4 and 5 .

We choose WS Small World and BA Scale-free network as the representative of uniform network, the number of nodes is the total number of people, and the average degree of network is $\bar{k}=6$. In order to avoid the influence of randomness on the simulation results, each simulation randomly selects one node from the network as the initial spreaders, and the rest as the ignorants.

Figure 3 shows the SIDRQ spreading of rumors on WS Small World and BA Scale-free network. We verify the stability of rumor-free equilibrium $\varphi_{0}$ in Theorem 4. Suppose $\quad \psi=0.00001, \quad \lambda=0.000004, \quad \mu=0.00000002$, $\alpha=0.00000002, \beta=0.0000002, \rho=0.0000002, \delta=0.002$, $\varepsilon=0.004, \gamma=0.00002$, and $\xi=0.0000001$. The basic number $R_{0} \approx 0.203326<1$ of system (1) is obtained through this calculation.

Figure 4 shows the SIDRQ spreading of rumors on WS Small World and BA Scale-free network. We verify the stability of $\varphi^{*}$ in Theorems 5. Suppose $\psi=0.0002$, $\lambda=0.000002, \mu=0.0000002, \alpha=0.0000002, \beta=0.0000002$, 
$\rho=0.0000002, \quad \delta=0.001, \quad \varepsilon=0.001, \quad \gamma=0.0002, \quad$ and $\xi=0.0000001$. The basic number $R_{0} \approx 7.499062>1$ of system (1) is obtained through the calculation (Figure 4 ).

\section{Conclusion}

This paper studies the dynamic characteristics of a new SIDRQ rumor-spreading model in a uniform network by adding a guidance and control mechanism. First, the basic reproduction number conditions for judging the existence of rumors are given. Secondly, the theoretical results of this paper show that when $R_{0}<1$, the rumor-free equilibrium $\varphi_{0}$ is globally asymptotically stable, and when $R_{0}>1$, rumorprevailing equilibrium $\varphi^{*}$ is locally asymptotically stable. In addition, through controllability analysis, it is clear that the system is controllable. Finally, numerical simulations verify the validity of the theoretical results. Next, our work is to study the optimal control problem of SIDRQ spreading of the rumors' model.

\section{Data Availability}

The data used to support the findings of the study are available within the article.

\section{Conflicts of Interest}

The authors declare that they have no conflicts of interest.

\section{Authors' Contributions}

All authors contributed equally to the writing of this paper. All authors read and approved the final manuscript.

\section{Acknowledgments}

This work was supported by the Science and Technology Program of Guizhou Province, under Grant nos. ZK[2021] 016 and [2016]1074 and Fund Project of Guizhou Minzu University, under Grant no. YJRCXM[2018]019.

\section{References}

[1] D. J. Daley and D. G. Kendall, "Stochastic rumours," IMA Journal of Applied Mathematics, vol. 1, no. 1, pp. 42-55, 1965.

[2] D. Maki and M. Thomson, Mathematical Models and Applications, Prentice-Hall, Englewood Cliff, NJ, USA, 1973.

[3] D. H. Zanette, "Criticality of rumor propagation on smallworld networks," Physical Review E, vol. 64, no. 5, Article ID 050901, 2001.

[4] D. H. Zanette, "Dynamics of rumor propagation on smallworld networks," Physical review E, Statistical, nonlinear, and soft matter physics, vol. 65, no. 41, pp. 041908-42126, 2002.

[5] Y. Moreno, M. Nekovee, and A. F. Pacheco, "Dynamics of rumor spreading in complex networks," Physical Review E-Statistical, Nonlinear and Soft Matter Physics, vol. 69, no. 2, pp. 279-307, 2003.

[6] M. Nekovee, Y. Moreno, G. Bianconi, and M. Marsili, "Theory of rumour spreading in complex social networks," Physica A:
Statistical Mechanics and Its Applications, vol. 374, no. 1, pp. 457-470, 2007.

[7] L. Wei, G. Jiao, and C. Xu, "Message spreading and forgetremember machanism on a scale-free network," Chinese Physics Letters, vol. 25, no. 6, pp. 2303-2306, 2008.

[8] L. Zhao, Q. Wang, J. Cheng, Y. Chen, J. Wang, and W. Huang, "Rumor spreading model with consideration of forgetting mechanism: a case of online blogging live journal," Physica A: Statistical Mechanics and Its Applications, vol. 390, no. 13, pp. 2619-2625, 2011.

[9] L. Zhao, J. Wang, Y. Chen, Q. Wang, J. Cheng, and H. Cui, "SIHR rumor spreading model in social networks," Physica A: Statistical Mechanics and Its Applications, vol. 391, no. 7, pp. 2444-2453, 2012.

[10] L. Zhao, X. Qiu, X. Wang, and J. Wang, "Rumor spreading model considering forgetting and remembering mechanisms in inhomogeneous networks," Physica A: Statistical Mechanics and Its Applications, vol. 392, no. 4, pp. 987-994, 2013.

[11] E. N. Gilbert, "Random graphs," The Annals of Mathematical Statistics, vol. 30, no. 4, pp. 1141-1144, 1959.

[12] P. Erdos and A. Renyi, "On random graphs I," Publicationes Mathematicae Debrecen, vol. 5, pp. 290-297, 1959.

[13] D. J. Watts and S. H. Strogatz, "Collective dynamics of 'small-world' networks," Nature, vol. 393, no. 6684, pp. 440-442, 1998.

[14] S. Wiggins, Introduction to Applied Nonlinear Dynamical Systems and Chaos, Springer, Berlin, Germany, 1990.

[15] P. Dreessche and J. Watmough, "Reproduction numbers and sub-threshold endemic equilibria for compartmental models of disease transmission," Mathematical Biosciences, vol. 180, pp. 29-48, 2002.

[16] T.-T. Zheng and L.-F. Nie, "Modelling the transmission dynamics of two-strain Dengue in the presence awareness and vector control," Journal of Theoretical Biology, vol. 443, pp. 82-91, 2018.

[17] S. Gershwin and D. Jacobson, "A controllability theory for nonlinear systems," IEEE Transactions on Automatic Control, vol. 16, no. 1, pp. 37-46, 1971. 